This is the pre-peer reviewed version of the following article:

Van Daele, S., \& Bernasco, W. (2012). Exploring directional consistency in offending: The case of residential burglary in The Hague. Journal of Investigative Psychology and Offender Profiling, 9(2), 135-148.

Which has been published in final form at: http://onlinelibrary.wiley.com/doi/10.1002/jip.1358/abstract

\title{
Exploring directional consistency in offending : The case of residential burglary in The Hague
}

\author{
Stijn Van Daele \\ Postdoctoral Fellow of the Research Foundation - Flanders (FWO), IRCP, \\ Ghent University \\ Wim Bernasco \\ Senior Researcher at the Netherlands Institute for the Study of Crime and Law \\ Enforcement (NSCR)
}

\begin{abstract}
Many aspects of human behavior are remarkably stable across times, places, and situations. Repetition and predictability also characterizes our geographical behavior. Prior research has confirmed that criminal behavior is no exception. Offenders tend to recidivate, and recidivists tend to be behaviorally consistent in many aspects, including geographical ones. The present study assesses directional consistency in offending. It reviews the literature on directional consistency. It proposes an improved measure of directional consistency, and empirically uses this measure to explore directional consistency amongst a set of 268 burglars in The Hague.
\end{abstract}

Keywords:

journey-to-crime $\cdot$ behavioral consistency $\cdot$ spatial analysis $\cdot$ directional statistics $\cdot$ burglary 


\section{Introduction}

Many aspects of human behavior are remarkably stable across times, places, and situations. This phenomenon has been called 'behavioral consistency' (Funder and Colvin 1991; Sherman, Nave, and Funder 2010). Behavioral consistency also applies to criminal behavior. Not only do many offenders recidivate, features of their target choices and modus operandi often recur in subsequent crimes (Davies 1992; Salfati and Alicia 2005; Tapper 2008; Woodhams, Hollin, and Bull 2007). For example, serial killers may repeatedly select victims with similar characteristics, robbers may repeatedly use the same type of weapon and burglars may repeatedly choose the same method of entry. Behavioral consistency can be practically useful in linkage analysis, an investigative activity aimed at assessing the likelihood that multiple offenses have involved the same offender (Tonkin, Woodhams, Bull, Bond, and Palmer 2011; Woodhams, Hollin, and Bull 2007).

Human geographical behavior is particularly stable and predictable. Research that followed the spatial tracks of a sample of mobile phone users concluded that "there is a potential $93 \%$ average predictability in user mobility, an exceptionally high value rooted in the inherent regularity of human behavior.” (Song, Qu, Blumm, and Barabasi 2010, p. 1021). Thus, if we know a person's whereabouts from hour to hour in the recent past, we can correctly predict their current whereabouts 93 percent of the time. The strong predictability of human geographic behavior raises the question whether this finding also holds for geographic offending behavior, in particular to the journey-to-crime. To what extent is the journey-tocrime characterized by consistency?

Three aspects of the journey may be characterized by consistency: destination, distance and direction. The present study focused on directional consistency, which can be defined as a serial offender's tendency to commit offenses at places that are located in the same direction from his or her anchor point (but not necessarily at the same distance). The objectives of the study were to review the prior literature on directional consistency in crime (section 2), to develop an improved measure of directional consistency (section 3) and a method to test it against random directionality (section 4), and to assess levels of directional consistency in a sample of residential burglars in The Hague, The Netherlands (sections 5 and 6). Section 7 discusses the findings against the background of what we know about the geography of crime and its perpetrators, including linkage analysis and geographic profiling.

\section{Journey-to-crime and directional consistency}

Offenders can display spatial consistency in their journey-to-crime. Like other studies that investigate the journey-to-crime, this study did not record actual travel behavior, but was confined to measuring the distance between the homes of offenders' and the locations of their offenses. As long as the offender's home is a fixed anchor point, three aspects of geographic 
offending behavior can be distinguished that may be characterized by consistency: destination, distance and direction.

The destination of an offense is the geographic location where it takes place. The distance is the Euclidian or straight-line distance between the offender's home and the destination. The direction is the angle between the home-offense line and a baseline reference (e.g. the equator). The destination is the most informative aspect, as it implies both the distance and the direction from home.

Destination consistency occurs if offenders repeatedly choose the same location to commit an offense. For example, a car thief may one week steal from a car in a parking lot, and return to the same parking lot to steal another car the week after. A street robber who attacked his previous victim at a train station may return to the same train station next time to rob another traveler. The repeat incident may represent a repeated attack against the same victim, but the essence of location consistency is that the offender returns to the same place where he offended before. Some research findings suggest destination consistency. Bernasco (2008) and Bowers \& Johnson (2004) demonstrate that the large majority of repeat and near repeat burglary victmizations are perpetrated by the same offenders who return to the locations of their prior burglaries, especially those repeat and near repeat victimizations that take place during the first days and weeks after the initial event. Additional support comes from case linkage studies that concluded that spatial and temporal proximity of offenses are better predictors for involvement of the same offender than other measures of behavioral consistency (Goodwill and Alison 2006; Tonkin et al. 2011).

Distance consistency occurs if offenders repeatedly commit offenses at approximately the same distance from their homes. Although there are many dozens of studies that address the home-offense distance, there are only three studies that actually investigate distance consistency in serial offenders (Smith, Bond \& Townsley, 2009; Townsley \& Sidebottom, 2010; Van Daele, 2010). The three studies are remarkably similar in their conclusion that offenders are much more distance consistent than had been assumed by those who interpreted the aggregated distance decay function as an individual phenomenon. Indicative of distance consistency, within-offender distance variation appears to be much smaller than betweenoffender distance variation.

Directional consistency is the third type of spatial consistency, and a central focus of the research described here. Directional consistency occurs if a serial offender repeatedly commits crimes at locations that are situated in the same direction from his or her main anchor point (typically the offender's home).

Clearly, destination consistency implies distance consistency as well as directional consistency, but distance consistency or directional consistency alone are not enough to imply destination consistency.

An extensive literature search identified four studies that address directional consistency. Lundrigan and Canter (2001) investigate the disposal site locations of serial murders. They find that murderers dispose the bodies of their victims precisely in the opposite direction from 
the preceding one. Goodwill \& Alison (2005) study serial murder, rape and burglary, and compare the angles between two subsequent offence sites targeted by the same criminal. They find the angle between two consequent murders being consequently larger than it is for rape and burglary. Both these studies use sequential angulation to define directional consistency. In sequential angulation only the angles between pairs of consecutive offenses are taken into account.

In two other studies, the directional consistency is calculated as the largest angle between any pair of crimes in the series. Kocsis et al. (2002) find evidence for directional consistency, because they establish that multiple burglaries lie in a rather narrow 'corridor'. Lundrigan and Czarnomski (2006) find similar results, demonstrating that the majority of offenders follow a rather narrow spatial corridor in their crime series. In sum, we found two different measures of directional consistency and mixed evidence for its existance.

One other study (Costanzo, Halperin, and Gale 1986) investigated whether criminals living nearby each other traveled in similar directions. This study is excluded here because it concerns the offense locations of different offenders, and not crime series involving the same offender.

In the next section we will argue that both measures of directional consistency that have been used in the literature - the average angle between pairs of consective offenses in a series, and the largest angle between any offense pair in a series - fails to fully capture the nature of directional consistency, and we will propose an improved measure.

\section{A new measure of directional consistency}

While directional data are not very common in the social sciences, they are frequently analysed in the natural and medical sciences like biology and geology. For example, they occur in bird migration research and in pollutant transport research in biology (e.g., Helbig 1991). In the social and behavioral sciences they sometimes appear in the form of circular data, in particular in the representations of daily, weekly, monthly or yearly temporal cycles. Directional and circular data have properties that require a separate branch of statistics called directional statistics or circular statistics (Fisher 1993; Jammalamadaka and SenGupta 2001; Mardia and Jupp 2000). For example, $0^{\circ}$ (or equivalently $360^{\circ}$ ) would suffice as a measure of central tendency for an angle of 2 degrees and one of 358 degrees, but $180^{\circ}$ would certainly not qualify (as it is approximately the opposite direction), although it is the mean of 2 and 358. Circular statitics suits circular data, but fortunately very little circular statistics is required to support our argument for an improved measure of directionality.

Of the four studies that have investigated directional consistency in offense series, two have measured directional inconsistency as sequential angulation (Lundrigan \& Canter, 2001; Goodwill \& Alison, 2005), and two others as maximal angulation (Lundrigan \& Czarnomsli, 2006; Kocsis et al. 2002). 
We believe both measures fail to capture directional consistency sufficiently, and propose an improved measure. Before describing the advantages of the new measure, we address the weaknesses of the sequential and the maximal angulation measures.

First, however, we discuss the definition of the 'angle' between two offenses, a definition that is used in all three measures. In all three measures, the angle between two offenses is defined by the offender's home (the vertex) and the two rays connecting the vertex to the two offenses. The angle between the offenses is defined to be always the absolute value of the smallest angle that can be formed by the three points. For example, in Figure 1 the circle labeled ' $H$ ' represents the offender's home, and the other two circles labeled ' 1 ' and ' 2 ' represent the locations of two offenses. Both $\alpha$ and $\beta$ form an angle between offenses 1 and 2 , but $\alpha$ is the smallest angle.

\section{( Insert Figure 1 here )}

Mathematically, the smallest angle between two offenses is calculated as follows using Cartesian geometry:

$\alpha=|| \tan ^{-1}\left(\frac{y^{2}-y^{0}}{x 2-x 0}\right)|-| \tan ^{-1}\left(\frac{y^{1}-y^{0}}{x 1-x_{0}}\right)||$

In which $\alpha$ is the angle expressed in radians, $(x 0, y 0)$ are the coordinates of the offender's home, and $(x 1, y 1)$ and $(x 2, y 2)$ are the coordinate pairs of the two offenses respectively. To convert radians to degrees, simply multiply by $180 / \pi$. Because most non-mathematicians are more familair with degrees than with radians, we will in the remainder express angular quantities in degrees.

To calculate sequential angulation in a series, one calculates the mean angle between all pairs of consecutive crimes. In a series of $n$ crimes, there are $n-1$ pairs of consecutive crimes.

\section{( Insert Figure 2 here )}

Consider, for example, panel 2a in Figure 2. The circle with an ' $\mathrm{H}$ ' at the centre represents the offender's home, the other circles represent the locations of the offenses committed by the offender, and the numbers represent the order in which they were committed. As four offenses were committed, there are three pairs of consecutive offenses (1 and 2, 2 and 3, and 3 and 4). All three pairs are $90^{\circ}$ apart, so that the average angle, the sequential angulation, equals $90^{\circ}$.

Panel $2 b$ in Figure 2 displays a different pattern, one in which the offender alternates between two directions only. This series also consists of four offenses, again all three consecutive pairs are $90^{\circ}$ apart, and therefore its sequential angulation measure also equals $90^{\circ}$.

The weakness of the sequential angulation measure is that it myopically focusses on the angles between consecutive offenses, and thereby ignores angles between non-consecutive offenses. As a result, the sequential angulation measure rates the patterns in panels $2 \mathrm{a}$ and $2 \mathrm{~b}$ both as $90^{\circ}$, despite the fact that offense pair 1 and 3, and offense pair 2 and 4 are perfectly 
directionally consistent (have zero angle) in panel $2 \mathrm{~b}$, but not in panel 2a. A better measure would rate the pattern in panel $2 \mathrm{~b}$ as more directionally consistent than the pattern in panel $2 \mathrm{a}$.

To calculate maximal angulation in a series, one calculates the largest angle between any pair of directions in the series.

Consider again panel 2a in Figure 2. The maximal angulation is $180^{\circ}$, which is the angle between offences 1 and 3, and also between offenses 2 and 4. Panel 2c displays a different pattern, in which offences 1, 2 and 4 are in the same direction, while offense 2 stands out because it is in the opposite direction. The maximal angulation of this pattern is also $180^{\circ}$, just as in panel 2a. The weakness of the maximal angulation measure is thus, that it myopically focuses on the largest angle, and ignores all other pairs of offenses. As a result, maximal angulation rates the patterns in panels $2 \mathrm{a}$ and $2 \mathrm{c}$ as equal, despite the fact that offense pairs 1$3,1-4$ and 3-4 are perfectly directionally consistent in panel 2c, but not in panel $2 \mathrm{a}$. A better measure of directional consistency would rate the pattern in panel $2 \mathrm{c}$ as more directionaly consistent than the pattern in panel $2 \mathrm{a}$.

To calculate the measure we propose, mean angulation, one calculates the average angle $\alpha_{\mathrm{av}}$ between all $n(n-1) / 2$ possible pairs of offenses in the series. In panel $2 \mathrm{a}$, mean angulation is $120^{\circ}$. This is the average of the angles between the offense pairs 1 and $2\left(90^{\circ}\right), 1$ and $3\left(180^{\circ}\right)$, 1 and $4\left(90^{\circ}\right), 2$ and $3\left(90^{\circ}\right), 2$ and $4\left(180^{\circ}\right)$, and 3 and $4\left(90^{\circ}\right)$. In panel $2 \mathrm{~b}$, mean angulation is $60^{\circ}$, the mean of pairs 1 and $2\left(90^{\circ}\right), 1$ and $3\left(0^{\circ}\right), 1$ and $4\left(90^{\circ}\right), 2$ and $3\left(90^{\circ}\right), 2$ and $4\left(0^{\circ}\right)$, and 3 and $4\left(90^{\circ}\right)$. In panel $2 \mathrm{c}$, it equals $90^{\circ}$, the average of the angles between the offense pairs 1 and $2\left(180^{\circ}\right), 1$ and $3\left(0^{\circ}\right), 1$ and $4\left(0^{\circ}\right), 2$ and $3\left(180^{\circ}\right), 2$ and $4\left(180^{\circ}\right)$, and 3 and $4\left(0^{\circ}\right)$. Mean angulation better captures directional consistency than both sequential angulation and maximal angulation. It is able to differentiate between the three patterns in Figures $2 a, 2 b$ and $2 \mathrm{c}$, wheres the two alternative measures can each only differentiate between two of the three patterns.

\section{Testing directional consistency}

Smaller mean angulation $\alpha_{\mathrm{av}}$ indicates greater directional consistency, as it implies that the offender tends to always travel in approximately the same direction to crime locations. Thus, directional consistency is maximal if $\alpha_{\mathrm{av}}=0$ and all offenses are located exactly in the same direction (but not necessarily at the same location). Directional consistency is minimal if $\alpha_{\mathrm{av}}=$ $180^{\circ}$, a situation that can only occur in a series of two crimes that are in opposite directions.

For any measured level of directional consistency, it may be necessary to test whether it differs significantly from the level of directional consistency that would be observed if the directions had been randomly chosen, i.e. if for every offense the offender randomly chooses a direction from the uniform distribution between 0 and $360^{\circ}$. In order words, in evaluating the specific pattern of angles in an offense series, a first indication of directional consistency is the test of whether $\alpha_{a v}$ is smaller than the expected value of $\alpha_{a v}$ under the $\mathrm{H}_{0}$ hypothesis of completely random directional choices. This test must take into account the number of 
offenses $n$ in the series, because the sampling distribution of $\alpha_{\mathrm{av}}$ under randomness depends on the number of offenses $n$ in the series.

Because in directional statistics many (sampling) distributions cannot be analytically derived, we used simulation to appoximate it. To calculate the sampling distribution of $\alpha_{\mathrm{av}}$ under random direction choice, for each offense series length $n$ between 2 and 25, we simulated 1 million series by first drawing random directions from a uniform distribution between $0^{\circ}$ and $360^{\circ}$, and subsequently calculating the average angle between the directions of all $n(n-1) / 2$ offense pairs. Figure 3 graphically displays the densities of these sampling distributions, with on the horizontal axis the angles expressed in degrees.

\section{( Insert Figure 3 here )}

For series of 2 offenses, this distribution is completely flat, because any angle between 0 and 180 degrees is equally likely, and angles lower than 0 and larger than 180 degrees cannot occur. For a series of 3 offenses, the minimum angle is again 0 degrees but the maximum is only 120 degrees, and the distribution is strongly right-skewed.

For each value of $n$, the expected value of $\alpha_{a v}$ is 90 degrees. Thus, in case of random direction choices the expected value of $\alpha_{\mathrm{av}}$ is always 90 degrees, independently of the number of offenses in the series. The standard error, however, decreases with $n$. The skewness also decreases with $n$, indicatng it is getting more right-skewed as $n$ increases. Using these simulated sampling distributions, it is easy to verify whether any particular observed $\alpha_{\mathrm{av}}$ should be considered as a significant level of directional consistency. The value of $\alpha_{a v}$ of a series of $n$ offenses should be amongst the 1 percent (or in case of a less conservative threshold, 5 percent) lowest $\alpha_{\mathrm{av}}$ values in the sampling distribution calculated for size $n$.

\section{( Insert Table 1 here )}

Table 1 lists for offense series of sizes 2-26 the number of offense pairs it contains, and the threshold values for significance (at $p<.05$ and $p<.01$ ). When a series comprises only two offences, significant directional consistency requires that $\alpha_{a v}<1.8^{\circ}$ (for $p<.01$ ) or $\alpha_{a v}<9^{\circ}$ (for $\mathrm{p}<.05$ ). For series if 15 crimes however, the directional consistency requires only that $\alpha_{\mathrm{av}}<72.36^{\circ}($ for $\mathrm{p}<.01)$ or $\alpha_{\mathrm{av}}<79.92^{\circ}$ (for $\left.\mathrm{p}<.05\right)$.

\section{Data}

Our dataset consists of a sample of police recorded and detected residential burglary series ( $n$ $>1$ ) in the Greater The Hague Area (that is served by the "Haaglanden" regional police force) in a seven year period (2001-2006). All burglary addresses and offender home addresses were geocoded on the basis of their 6-digit postal code. The 6-digit postal code is a fairly small area containing approximately 20 residential properties on average (e.g. one side of a small street segment, or part of a terraced multi-household dwelling). The centroid of this postal code area was assigned to the burglary targets and burglars' homes located inside it. 
An additional selection criterion applied was, that only offenders were included who had not changed their residence during the series. This selection was applied because moving causes a change in the anchor point of the directional consistency calculations. Although in principle an acceptable alternative would have been to split these series into a part 'before the move' and another part 'after the move', this would have resultated in a double hierachical data structure (offenses nested in series which are nested in offenders) and associated analytical complications. We decided to exclude them for two reasons. Theoretically, it may be expected that when offenders move, their spatial awareness changes in complex ways that are difficult to model without knowledge of their old and new routine activities (Bernasco 2010). For example, they may still attend the same school as before, or shift to another school. Statistically, the increased number of observations that would be won by including movers would not outweigh the increased statistical complexities of modeling three hierachical levels.

The result is a dataset of 268 burglars each of whom had been involved in two or more burglaries, and for whom measures of directional consistency can be calculated. Together the 268 burglars committed 1116 detected residential burglaries. The crimes committed by each offender vary between 2 and 26. Table 2 specifies how many offenses had been committed by how many offenders.

( Insert Table 2 here )

\section{Findings}

The present section applies the improved measure of directional consistency in the The Hague burglary series data. Figure 4 displays a histogram of the distribution of $\alpha_{a v}$ in the sample, where each bar represents a width of 10 degrees. The shaded parts of the bars represent the proportion of the cases for which $\alpha_{a v}$ is statistically significant $(p<.01)$ whereas the unshaded parts represent $\alpha_{\mathrm{av}}$ values that are, athough in the same range of magnitude, nonsignificant (these are generaly the shorter burglary series).

( Insert Figure 4 here )

Close examination of Figure 4 suggests two groups of offenders. First, a substantial proportion of the offenders has a strong directional consistency $\left(\alpha_{\mathrm{av}}<20^{\circ}\right)$. Another substantial part shows $\alpha_{a v}$ values between $30^{\circ}$ and $80^{\circ}$, a more moderate form of directional consistency.

Clearly, $\alpha_{\mathrm{av}}$ and significance are related but the relation is confounded by the numbers of crimes in the series. Table 3 presents an alternative view of the relation. For each series size, it presents the number of offense pairs involved (i.e., $n(n-1) / 2$ ), the threshold value necessary for significance at $\mathrm{p}<.01$, and the number and percentage of series that have an $\alpha_{\mathrm{av}}$ value below the threshold. The last three columns are repeated for $\mathrm{p}<.05$. 
In general, 98 out of 268 burglars $(36.6 \%)$ demonstrate a significant $(\mathrm{p}<.01)$ level of directional consistency, 140 burglars $(52.2 \%)$ have directional consistency at significance level $\mathrm{p}<.05$. This shows that many offending patterns are characterized by directional consistency.

Next, two correlations are considered, as one may expect them to influence offenders' directional consistency. First, the correlation between directional consistency and size of the crime series is considered. We observe no substantial correlation $(r=.08)$ between the number of crimes in a series an offender's directional consistency.

We do find a slight negative correlation $(r=-.23)$ between the average home-offense distance in a crime series and its mean angulation $\alpha_{\mathrm{av}}$. Thus, offenders who perpetrate further from their anchor point are more directionally consistent and have a more narrow 'corridor' to their crime locations than those who perpetrate closer to home. Our calculation of $\alpha_{a v}$ makes no reference to distance. Still, we find a relationship, be it an inverse one. This means that offenders' operational ranges (the distances between their two widest offences) may be similar and independent of the distance they travel.

In general, with a demarcation criterion of $\mathrm{p}<.01,98$ out of 268 burglars $(36.6 \%)$ are directionally consistent. When a criterion of $\mathrm{p}<.05$ is taken, 140 burglars $(52.2 \%)$ display directional consistency. This shows that within reasonable margins of likelihood, a considerable number of offense series, but certainly not all, are characterized by directional consistency. Table 2 represents the amount of significant directionally consistent patterns in relation to the numbers of offenses in the series and the number of offense pairs that the measure is based on.

\section{Discussion}

Proposing a new measure for calculating directional consistency in offence series, we find two main groups of offenders. A first offender type has a very strong directional bias i.e. a small average angle between his offence pairs. One third of offenders $(36.6 \%)$ has a pattern with a directional consistency at significance level $\mathrm{p}<.01$. A second group of offenders has a directional consistency between $35^{\circ}$ and $70^{\circ}$, close the highest possible maximum.

The results are quite in similar to what Lundrigan and Czarnomski (2006) have found. Using the angle between the two widest offences, they also found a large proportion of offenders operating in a corridor of less than $45^{\circ}$ and a second group of offenders operating in a wider corridor of between $136^{\circ}$ and $180^{\circ}$. As they only use the angle between the two widest offences and the method in the present paper calculates the average angle between all offence pairs, the exact sizes of the angles are obviously different. Yet, the existence of both groups is largely the same. 
Such findings are largely in line with the marauder-commuter dichotomy as developed by Canter and also tested by others (Canter 2000; Canter and Alison 2000; Canter and Gregory 1994; Canter and Larkin 1993; Canter and Youngs 2008; Meaney 2004; Paulsen 2007). On the one hand there are offenders who are characterized by a high level of directional consistency. As all their offences are located at the same side of their anchor point, they are classified as commuters. On the other hand, offenders with weak directional consistency commit crimes in many different directions around their anchor point, and are therefore classified as marauders.

Through calculating average angles, our measure of directional consistency is mathematically independent from distance. Consequently, we expect no relationship between average travelled distance and directional consistency. Empirically, however, it is found that that larger distance is positively related to directional consistency (i.e. to smaller $\alpha_{\mathrm{av}}$ ). First of all, this means that directional consistency measures another component of the journey to crime than distance does. Second, it demonstrates that the mean angulation does not increase with distance. Such a finding may be interpreted to support the concept of awareness space, as developed by Brantingham and Brantingham (1981; 1993). In this concept, certain activity areas - work, leisure,... - are called nodes and it is assumed that offenders operate in the area that covers such nodes. The area around a certain node may be consistent (e.g. visual range), independent of how far this node is located from the offender's residence. Figure 5 demonstrates that such an assumption makes the angle smaller if offenders operate further away from home (right picture), than closer to home (left picture).

\section{( Insert Figure 5 here )}

In this picture, two crimes are committed each at one extreme side of the awareness space node (presented by the grey circle). The crime trips are referred to as the white arrows. Although the size of the awareness space remains the same, the angle formed by the location of both crimes and the home is much smaller - i.e. strong directional consistency - in the right situation than it is for the left one. Thus, assuming that the area around certain activity nodes remains consistent, larger travelled distances lead to a smaller angle and, hence, stronger directional consistency.

We demonstrate that a substantive proportion of offenders has directional consistency in their pattern. Over one third of offenders have a directional consistency that is stronger than the first percentile of randomly generated patterns $(\mathrm{p}<.01)$. If we loosen this threshold to the lower fiver percent of randomly generated patterns $(\mathrm{p}<.05)$, more than half of the burglars in our sample demonstrate such consistency.

A few caveats concerning the present study have to be mentioned. First, the sample used is rather small and only considers burglars. Therefore, more extensive and future research on directional consistency may include larger samples and different crime types. Second, cut values to judge whether patterns demonstrate significant directional consistency vary with the size of the crime series. This makes it less straightforward to judge the significance level of a certain pattern (see also figure 4): one needs to calculate the random pattern first and only in a later stage can the empirical pattern be compared to this pattern. Third - and this is relevant 
for every research in this area - we only work with caught offenders. If we consider it possible that more mobile offenders are less likely to get caught (Eck and Weisburd 1995; McIver 1981), it may as well be that offenders operating on a smaller area - and thus showing stronger directional bias - get caught more easily. Fourth, the present study did not take into account the presence of potential mobility frictions other than distance. For example, although the study area generally consists of flat land with a good mobility infrastructure for travel by foot, bicyle, motor bicycle, car and public transport, for some anchor points directionality is restricted by natural (e.g. the seashore) or constructed (e.g. railway and highways that obstruct cross-traffic) barriers.

Nevertheless, we have demonstrated that a large percentage of offending patterns show significant levels of directional consistency. Therefore, and taking into account the fact that directionality is an underrepresented area in the study of crime trips, this paper demonstrates the usefulness and importance of directionality research. We hope to see future journey-tocrime studies take their analysis a step further in this direction.

\section{References}

Bernasco, Wim. 2010. "A Sentimental Journey to Crime; Effects of Residential History on Crime Location Choice." Criminology 48:389-416.

Bowers, Kate J. and Shane D. Johnson. 2004. "Who Commits Near Repeats? A Test of the Boost Explanation." Western Criminology Review 5:12-24.

Brantingham, Patricia and Paul Brantingham. 1981. "Notes on the geometry of crime." Pp. 27-54 in Environmental criminology, edited by P. Brantingham and P. Brantingham. Beverly Hills: Sage.

- 1993. "Nodes, paths and edges: considerations on the complexity of crime and the physical environment." Journal of Environmental Psychology 13:3-28.

Canter, David. 2000. "Offender profiling and criminal differentiation." Legal and Criminological Psychology 5:23-46.

Canter, David and Laurence J. Alison. 2000. "Profiling property crimes." Pp. 318 in Offender Profiling Series, edited by D. Canter. Aldershot: Ashgate.

Canter, David and A. Gregory. 1994. "Identifying the residential location of serial rapists." Journal of the Forensic Sciency Society 34:169-175.

Canter, David and Paul Larkin. 1993. "The environmental range of serial rapists." Journal of Environmental Psychology 13:63-69.

Canter, David and Donna Youngs. 2008. "Geographical offender profiling: origins and principles." Pp. 1-18 in Principles of geographical offender profiling, Psychology, crime and law, edited by D. Canter and D. Youngs. Aldershot: Ashgate.

Costanzo, Michael, William Halperin, and Nathan Gale. 1986. "Criminal mobility and the directional component in journeys to crime." Pp. 73-95 in Metropolitan crime patterns, edited by R. Figlio, S. Hakim, and G. Rengert. Monsey: Criminal Justice Press.

Davies, Anne. 1992. "Rapists' behaviour: a three aspect model as a basis for analysis and the identification of serial crime." Forensic Science International 55:173-194.

Eck, John and David Weisburd. 1995. "Crime places in crime theory." Pp. 1-33 in Crime and place, Crime prevention studies, edited by J. Eck and D. Weisburd. Monsey: Criminal Justice Press. 
Fisher, N.I. 1993. Statistical Analysis of Circular Data. Cambridge: Cambridge University Press.

Funder, David and Randall Colvin. 1991. "Explorations in behavioral consistency: properties of person, situations, and behaviors." Journal of Personality and Social Psychology 60:773-794.

Goodwill, Alasdair M. and Laurence J. Alison. 2006. "The development of a filter model for prioritising suspects in burglary offences." Psychology, Crime \& Law 12:395-416.

Goodwill, Alasdair. M. and Laurence. J. Alison. 2005. "Sequential angulation, spatial dispersion and consistency of distance attack patterns from home in serial murder, rape and burglary." Psychology Crime \& Law 11:161-176.

Helbig, Andreas. 1991. "Inheritance of migratory direction in a bird species: a cross-breeding experiment with SE- and SW-migrating blackcaps (\&lt;i\&gt;Sylvia atricapilla\&lt;/i\&gt;)." Behavioral Ecology and Sociobiology 28:9-12.

Jammalamadaka, S. Rao and S SenGupta. 2001. Topics in Circular Statistics, vol. 5. Singapore: World Scientific.

Kocsis, Richard, Ray Cooksey, Harvey Irwin, and Greg Allen. 2002. "A further assessment of 'circle theory' for geographic psychological profiling." Australian and New Zealand Journal of Criminology 35:43-62.

Lundrigan, Samantha and David Canter. 2001. "A multivariate analysis of serial murderers' disposal site location choice." Journal of Environmental Psychology 21:423-432.

Lundrigan, Samantha and Sarah Czarnomski. 2006. "Spatial characteristics of serial sexual assault in New Zealand " Australian and New Zealand Journal of Criminology 39:218-231.

Mardia, K.V. and P.E. Jupp. 2000. Directional Statistics. Chichester: Wiley.

McIver, John. 1981. "Criminal mobility: a review of empirical studies." Pp. 20-47 in Crime spillover, edited by S. Hakim and G. Rengert. Beverly Hills: Sage.

Meaney, Rebecca. 2004. "Commuters and marauders: an examination of the spatial behaviour of serial criminals." Journal of Investigative Psychology and Offender Profiling 1:121137.

Paulsen, Derek. 2007. "Improving geographic profiling through commuter/marauder prediction." Police Practice and Research 8:347-357.

Salfati, C. Gabrielle and L. Bateman Alicia. 2005. "Serial homicide: an investigation of behavioural consistency." Journal of Investigative Psychology and Offender Profiling 2:121-144.

Sherman, Ryne A., Christopher S. Nave, and David C. Funder. 2010. "Situational similarity and personality predict behavioral consistency." Journal of Personality and Social Psychology 99:330-343.

Song, Chaoming, Zehui Qu, Nicholas Blumm, and Albert-Laszlo Barabasi. 2010. "Limits of Predictability in Human Mobility." Science 327:1018-1021.

Tapper, Sarah. 2008. "Testing the assumption of behavioural consistency in a New Zealand sample of serial rapists." Philosophy, Victoria University, Wellington.

Tonkin, Matthew, Jessica Woodhams, Ray Bull, John W. Bond, and Emma J. Palmer. 2011. "Linking Different Types of Crime Using Geographical and Temporal Proximity." Criminal Justice and Behavior 38:1069-1088.

Woodhams, Jessica, Clive R. Hollin, and Ray Bull. 2007. "The psychology of linking crimes: A review of the evidence." Legal and Criminological Psychology 12:233-249. 
Figure 1: Smallest angle $(\alpha)$ and largest angle $(\beta)$ between two offenses

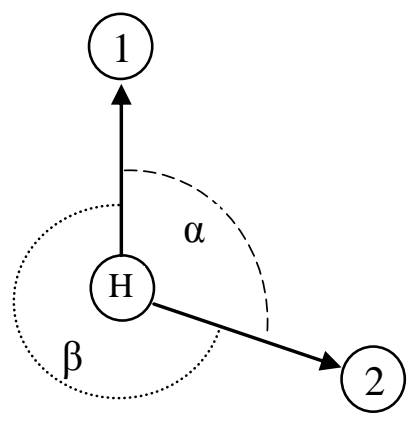

Figure 2: Sequential, Maximal and Mean Angulation in Three Crime Series
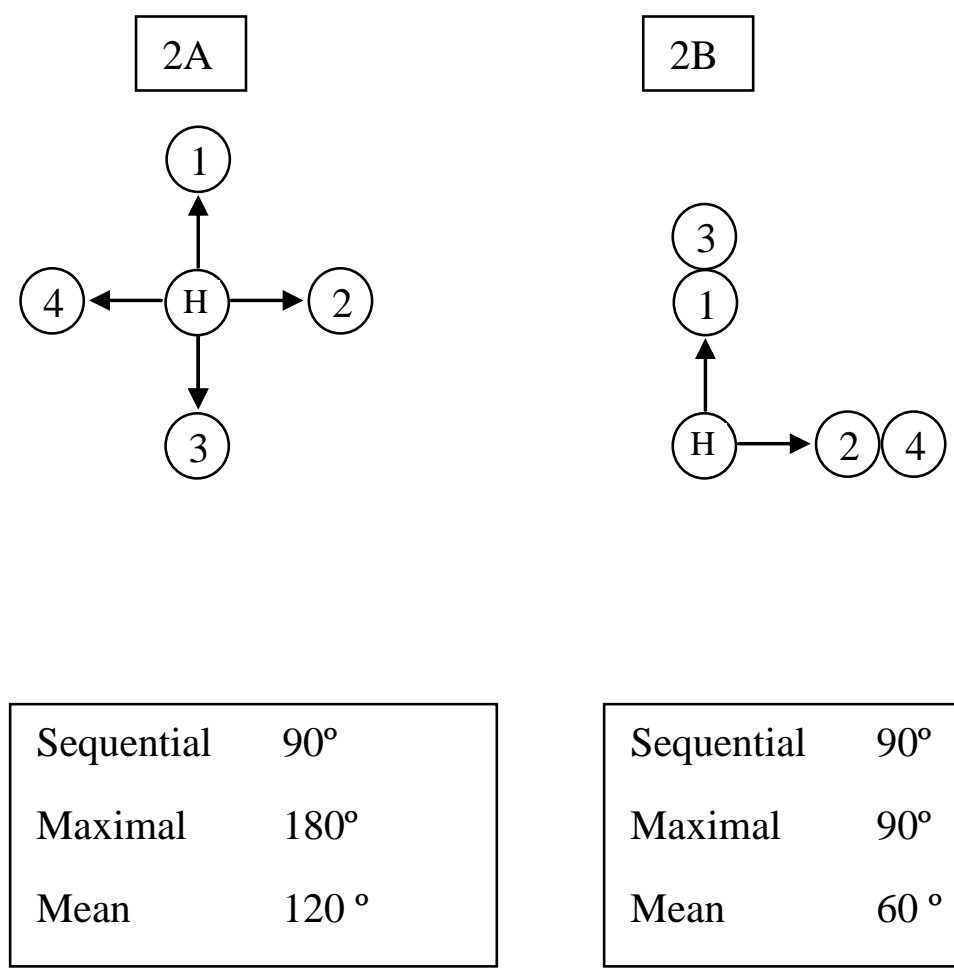

\section{$2 \mathrm{C}$}
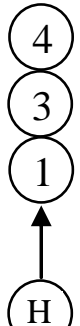

(H)

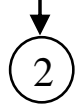

(2)

\begin{tabular}{|ll|}
\hline Sequential & $90^{\circ}$ \\
Maximal & $90^{\circ}$ \\
Mean & $60^{\circ}$ \\
\hline
\end{tabular}

\begin{tabular}{|ll|}
\hline Sequential & $120^{\circ}$ \\
Maximal & $180^{\circ}$ \\
Mean & $90^{\circ}$
\end{tabular}


Figure 3: Distributions of mean angulation of crime series under uniform randomization of angles, by size of the crime series
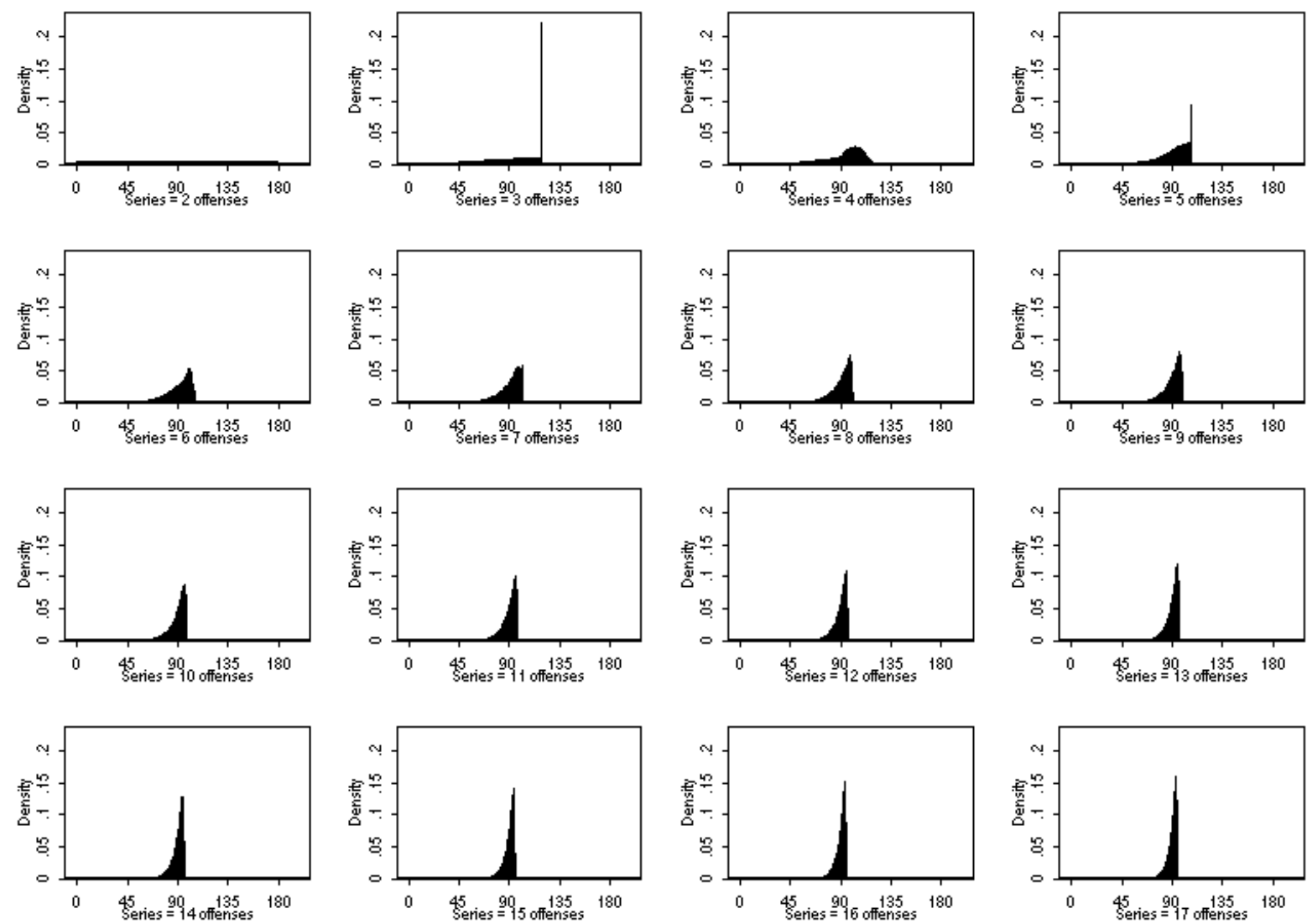
Figure 4: Directional consistency and significant directional consistency

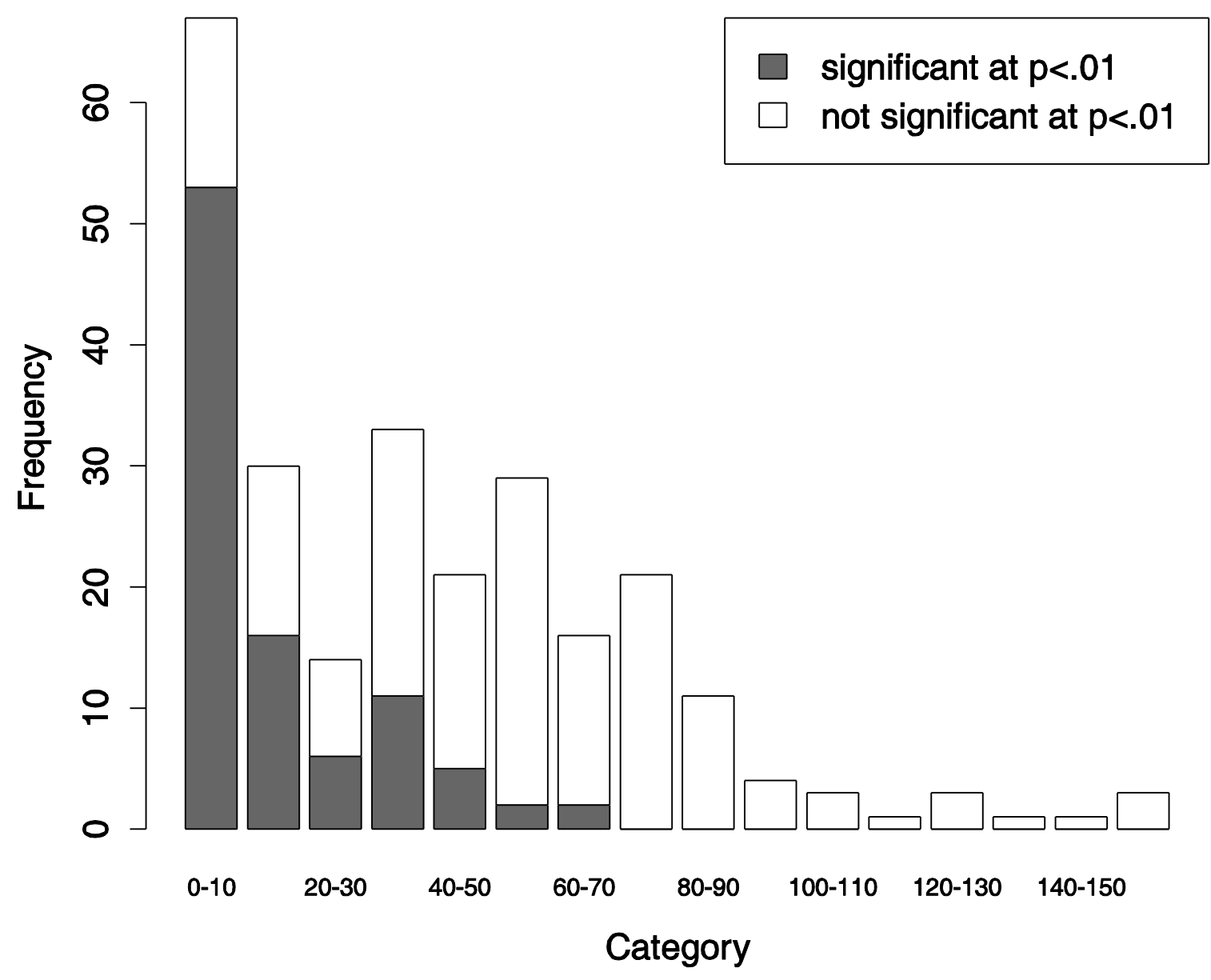

Figure 5: Directional consistency related to distance
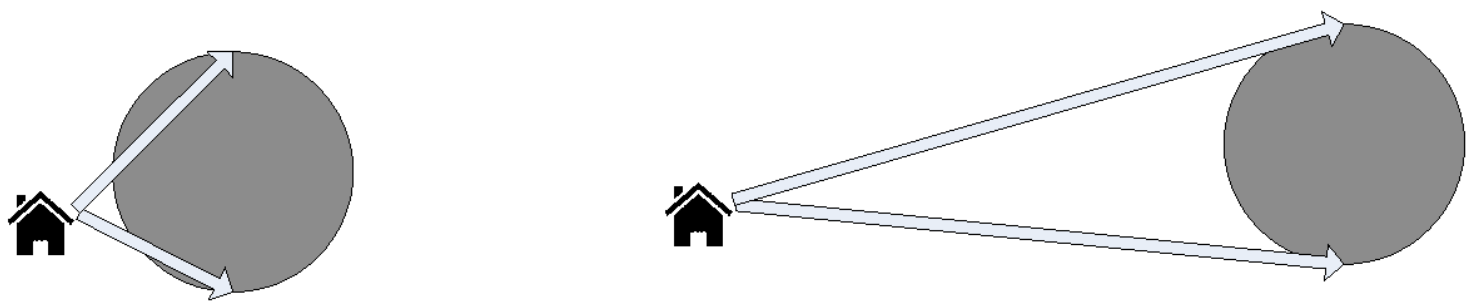
Table 1: Significance thresholds (in degrees) for testing against random directionality. Simulation results. Read example (row 3): A series of 4 offenses involves 6 pairwise comparisons, if the average angle across those 6 pairs is below 45.9 degrees there is significant directional consistency at $p<.05$, if it is below 26.6 degrees there is significant directional consistency at $\mathrm{p}<.01$.

\begin{tabular}{|c|c|c|c|}
\hline $\begin{array}{c}\# \text { offences } \\
(n)\end{array}$ & $\begin{array}{c}\text { \# offence pairs } \\
n(n-1) / 2\end{array}$ & $\mathrm{p}<.05$ & $\mathrm{p}<.01$ \\
\hline 2 & 1 & $9.0^{\circ}$ & $1.8^{\circ}$ \\
\hline 3 & 3 & $31.0^{\circ}$ & $13.9^{\circ}$ \\
\hline 4 & 6 & $45.9^{\circ}$ & $26.6^{\circ}$ \\
\hline 5 & 10 & $55.8^{\circ}$ & $37.4^{\circ}$ \\
\hline 6 & 15 & $62.5^{\circ}$ & $45.4^{\circ}$ \\
\hline 7 & 21 & $67.0^{\circ}$ & $51.5^{\circ}$ \\
\hline 8 & 28 & $70.2^{\circ}$ & $56.5^{\circ}$ \\
\hline 9 & 36 & $72.5^{\circ}$ & $60.1^{\circ}$ \\
\hline 10 & 45 & $74.3^{\circ}$ & $63.4^{\circ}$ \\
\hline 11 & 55 & $76.0^{\circ}$ & $65.7^{\circ}$ \\
\hline 12 & 66 & $77.2^{\circ}$ & $67.9^{\circ}$ \\
\hline 13 & 78 & $78.1^{\circ}$ & $69.7^{\circ}$ \\
\hline 14 & 91 & $79.0^{\circ}$ & $71.1^{\circ}$ \\
\hline 15 & 105 & $79.9^{\circ}$ & $72.4^{\circ}$ \\
\hline 16 & 120 & $80.5^{\circ}$ & $73.4^{\circ}$ \\
\hline 17 & 136 & $81.0^{\circ}$ & $74.3^{\circ}$ \\
\hline 18 & 153 & $81.5^{\circ}$ & $75.2^{\circ}$ \\
\hline 19 & 171 & $82.1^{\circ}$ & $76.0^{\circ}$ \\
\hline 20 & 190 & $82.4^{\circ}$ & $76.7^{\circ}$ \\
\hline 21 & 210 & $82.8^{\circ}$ & $77.4^{\circ}$ \\
\hline 22 & 231 & $83.2^{\circ}$ & $77.9^{\circ}$ \\
\hline 23 & 253 & $83.5^{\circ}$ & $78.5^{\circ}$ \\
\hline 24 & 276 & $83.7^{\circ}$ & $79.0^{\circ}$ \\
\hline 25 & 300 & $84.1^{\circ}$ & $79.4^{\circ}$ \\
\hline 26 & 325 & $84.2^{\circ}$ & $79.7^{\circ}$ \\
\hline
\end{tabular}


Table 2: Distribution of serial offenders and offenses (empirical data)

\begin{tabular}{rrrrr}
\hline $\begin{array}{r}\text { \# offenses } \\
\text { per offender }\end{array}$ & \# offenders & \% offenders & \# offenses & \% offenses \\
\hline 2 & 107 & 39.93 & 214 & 19.18 \\
3 & 46 & 17.16 & 138 & 12.37 \\
4 & 34 & 12.69 & 136 & 12.19 \\
5 & 24 & 8.96 & 120 & 10.75 \\
6 & 23 & 8.58 & 138 & 12.37 \\
7 & 12 & 4.48 & 84 & 7.53 \\
8 & 2 & 0.75 & 16 & 1.43 \\
9 & 4 & 1.49 & 36 & 3.23 \\
10 & 2 & 0.75 & 20 & 1.79 \\
11 & 3 & 1.12 & 33 & 2.96 \\
12 & 1 & 0.37 & 12 & 1.08 \\
13 & 3 & 1.12 & 39 & 3.49 \\
14 & 1 & 0.37 & 14 & 1.25 \\
15 & 1 & 0.37 & 15 & 1.34 \\
16 & 1 & 0.37 & 16 & 1.43 \\
17 & 1 & 0.37 & 17 & 1.52 \\
19 & 1 & 0.37 & 19 & 1.70 \\
23 & 1 & 0.37 & 23 & 2.06 \\
26 & 1 & 0.37 & 26 & 2.33 \\
\hline Total & 268 & 100 & 1116 & 100 \\
\hline
\end{tabular}

Source: Police registration, Haaglanden Police Force

Table 3: Significant directional consistency in relation to crimes.

\begin{tabular}{llllllll}
\hline \multirow{2}{*}{ Crimes } & $\mathrm{n}$ & \multicolumn{2}{l}{$\mathrm{P}<.01$} & \multicolumn{3}{l}{$\mathrm{P}<.05$} \\
\cline { 2 - 7 } & & value & $\mathrm{n}$ & $\%$ & value & $\mathrm{n}$ & $\%$ \\
\hline 2 & 107 & $1.76^{\circ}$ & 31 & 29.0 & $8.96^{\circ}$ & 45 & 42.1 \\
3 & 46 & $13.82^{\circ}$ & 13 & 28.3 & $30.98^{\circ}$ & 18 & 39.1 \\
4 & 34 & $26.66^{\circ}$ & 11 & 32.4 & $45.88^{\circ}$ & 13 & 38.2 \\
5 & 24 & $37.37^{\circ}$ & 6 & 25.0 & $55.84^{\circ}$ & 17 & 70.8 \\
6 & 23 & $45.41^{\circ}$ & 12 & 52.2 & $62.46^{\circ}$ & 17 & 73.9 \\
7 & 12 & $51.52^{\circ}$ & 6 & 50.0 & $67.00^{\circ}$ & 10 & 83.3 \\
$8+$ & 22 & varying & 19 & 86.4 & varying & 20 & 90.9 \\
\hline Total & 268 & varying & 98 & 36.6 & varying & 140 & 52.2 \\
\hline
\end{tabular}

